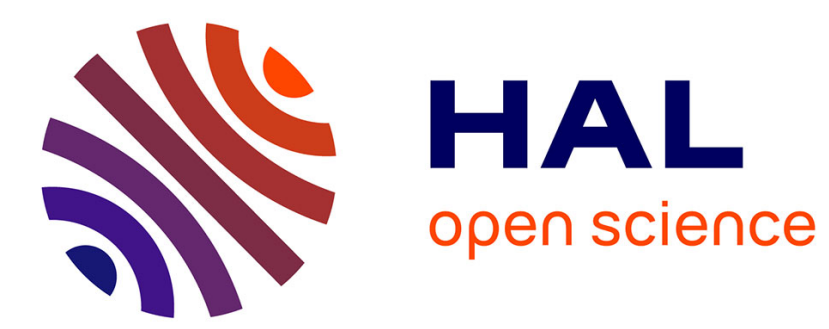

\title{
On the Unified Micromechanics Constitutive Description of One-Way and Two-Way Shape Memory Effects
}

\author{
Q.-P. Sun, C. Lexcellent
}

\section{To cite this version:}

Q.-P. Sun, C. Lexcellent. On the Unified Micromechanics Constitutive Description of One-Way and Two-Way Shape Memory Effects. Journal de Physique IV Proceedings, 1996, 06 (C1), pp.C1-367-C1375. 10.1051/jp4:1996135 . jpa-00254167

\section{HAL Id: jpa-00254167 https://hal.science/jpa-00254167}

Submitted on 1 Jan 1996

HAL is a multi-disciplinary open access archive for the deposit and dissemination of scientific research documents, whether they are published or not. The documents may come from teaching and research institutions in France or abroad, or from public or private research centers.
L'archive ouverte pluridisciplinaire HAL, est destinée au dépôt et à la diffusion de documents scientifiques de niveau recherche, publiés ou non, émanant des établissements d'enseignement et de recherche français ou étrangers, des laboratoires publics ou privés. 


\title{
On the Unified Micromechanics Constitutive Description of One-Way and Two-Way Shape Memory Effects
}

\author{
Q.-P. Sun $(1)$ and C. Lexcellent* \\ Department of Engineering Mechanics, Tsinghua University, Beijing 100084, P.R. China \\ * Laboratoire de Mécanique Appliquée, Université de Franche-Comté, 25030 Besançon, France
}

\begin{abstract}
A micromechanics constitutive model for the two-way shape memory effect (TWSME) of single crystal shape memory alloys is proposed to describe the TWSME behavior during the cooling and heating process after some training of the alloys. The model is established on two physical bases: (1) the micromechanics crystallographic constitutive theory of single crystal recently developed by SUN et al.[3] and (2) the analysis of the stored elastic strain energy due to dislocations that are produced in the training process of a single kind of variant and the analysis of the interactions between the stress fields of dislocations and transformations. Theoretical predictions of the model are in agreement with the available experimental observations. Finally the present model can be used as a theoretical basis to predict the TWSME of polycrystals.
\end{abstract}

\section{INTRODUCTION}

It is well known [1-2] that the two-way shape memory effect (TWSME) is associated with the forward $(\mathrm{p} \rightarrow \mathrm{m})$ and reverse $(\mathrm{m} \rightarrow \mathrm{p})$ transformations of preferentially oriented martensites under thermal cycles. Generally it is thought that the preferred martensites are formed on cooling by residual stress fields or dislocation structures that are introduced by thermomechanical treatment or training process. Dislocations can be introduced in shape memory alloys in several ways: (1) during crystal growth and quenching procedure, (2) by pure plastic deformation of the parent or martensitic phases, (3) by transformation cycling either spontaneously or under applied stresses. The last one is usually employed by the material scientists to obtain TWSME and is often termed as the "training process" or "education". The existance of a given set of dislocation structures (and correspondingly the generated internal stress field), which is related with the formation of a specific kind of martensite variants during the training process, will change or modify the relative stability between the parent and martensite phases and the relative stability among different kinds of martensite variants. For example, it will produce a shift in the $\mathbf{M}_{\mathrm{s}}$ temperature and will make some variants thermodynamically more favorable than others thus leading to TWSME.

A lot of micromechanical [3-7] and phenomenological [8-10]constitutive studies have been performed to describe the constitutive behavior of shape memory alloys, so far some important macroscopic behavior in shape memory alloys such as pseudoelasticity, shape memory effect, ferroelasticity, rubber-like elasticity, etc. can be, to some extent, quantitatively modelled. However, almost all these descriptions have the limitations in the sense that the prediction of the two-way memory effect are not available by these models. This is due to the fact that all these models are originally intended only for the one-way shape memory effect (OWSME). Patoor et al. [11] first studied the internal stress effect in TWSME and established a phenomenological flow rule and criterion to describe this typical behavior. With the progress of investigation, to establish a micromechanics and mechanism based constitutive model to include both OWSME and TWSME in an unified manner seems to be both necessary and possible.

\footnotetext{
${ }^{\text {(1) }}$ Present address: Department of Mechanical Engineering, the Hong Kong University of Science and Technology, Clear Water Bay, Kowloon, Hong Kong.
} 
The purpose of the present paper is to perform a micromechanics based study on the unified constitutive prediction of both OWSME and TWSME phenomena. The investigation is based upon the micromechanics crystallographic constitutive model of single crystals recently developed by SUN et al.[3] and based upon the analysis of the stored elastic strain energy due to dislocations and the analysis of the interaction effect between the stress fields of dislocations and transformations. In the present study the TWSME is considered as a special type of previous loading history effect (related only to the dislocations) and is quantitatively incorperated into the transformation (yielding) condition. This effect is also termed as the coupling effect between classical plasticity and purely transformation induced plasticity (such as those in TRIP steels). In section 2, the proposed constitutive model is formulated and the transformation conditions capable of describing both the TWSME and OWSME are discussed. The conclusion is finally summarized in section 3 .

\section{THE MICROMECHANICS CONSTITUTIVE MODEL}

To establish a micromechanics based transformation constitutive model, a representative material sample (constitutive element) is taken from a single crystalline bulk as the subject of study. A uniform temperature (T) distribution is assumed in the element and the external macroscopic stress $\Sigma$ or strain $E$ is applied on the boundary. In the case of OWSME, the initial state of the element (before transformation) is constituted by parent phase only without dislocation, wherease in the case of TWSME, after some training process the initial state of the element is constituted by parent phase with dislocation structures that are related to the formation of some particular kind of martensite variants during the training. In this case some amount of elastic strain energy due to the internal stress of dislocations will be stored in the element. With the change of temperature $T$ (such as cooling) or application of stress or strain, the forward (or reverse) transformation may happen. Some kinds of martensitic variants (For example, 24 in alloys with cubic lattice structure) may appear and growth (or shrink and vanish). In the case of reorientation process, the differently oriented martensite variants will coalesce.

\subsection{Kinematical relations of transformation plasticity and classical plasticity}

Starting from the crystallographic theory of martensitic transformation we can calculate the transformation strain $\varepsilon_{\mathrm{s}}^{\mathrm{tp}}$ (here "tp" represents "transformation plasticity") of the sth kind of variants in a single crystal as

$$
\varepsilon_{s}^{t p}=g \mathbf{R}_{s}=\frac{1}{2} g\left(\mathbf{e}_{s} \mathbf{n}_{s}+\mathbf{n}_{s} \mathbf{e}_{s}\right),
$$

where $\mathbf{R}_{s}$ is called the orientation tensor of the sth kind of martensite variant (habit plane variant), $\mathbf{e}_{\mathbf{s}}$ is the displacement direction of the invariant plane of the sth kind of variant, $\mathbf{n}_{\mathbf{s}}$ is the corresponding normal of the invariant plane and $g$, usually taken as a material constant, is the displacement magnitude of the invariant plane per unit length along the normal $\mathbf{n}_{s}$.

Denote the volume occupied by the $s$ th kind of variants $(s=1, \ldots, N, N$ is the total kinds of variants) by $V_{s}$ and the corresponding volume fraction by $f_{s}\left(=V_{s} / V\right)$. The total volume of transformed variants, its volume fraction and the parent phase's volume are $V_{i}=\sum_{s=1}^{N} V_{s}, f=\sum_{s=1}^{N} f_{s}$ and $V_{p}=V-V_{i}$, respectively. We can derive the relation between the microscopic strain $\varepsilon$ and macroscopic strain $\mathrm{E}$ (here the strains are assumed to be small and can therefore be decomposed into elastic and plastic parts):

$$
\mathrm{E}=\left\langle\varepsilon^{e}\right\rangle_{V}+\left\langle\varepsilon^{p}\right\rangle_{V}=\mathrm{E}^{e}+\mathrm{E}^{p}=\mathrm{M}: \Sigma+\mathrm{E}^{p},
$$

where $<>$ denotes volume average and the thermal strain and stress are neglected for simplicity. By the way we have also assumed that the martensite and the parent phase have the same elastic compliance tensor $\mathbf{M}$. 
According to the crystallographic theory of martensitic transformation, we further have

$$
\begin{aligned}
\mathrm{E}^{t p} & =\left\langle\varepsilon^{t p}\right\rangle_{V}=f\left\langle\varepsilon^{t p}\right\rangle_{V_{i}}=\sum_{s=1}^{N} f_{s} \varepsilon_{s}^{t p}=g \sum_{s=1}^{N} f_{s} \mathbf{R}_{s} . \\
\dot{\mathrm{E}}^{t p} & =\sum_{s=1}^{N} \dot{f}_{s} \varepsilon_{s}^{t p}=g \sum_{s=1}^{N} \dot{f}_{s} \mathbf{R}_{s}
\end{aligned}
$$

The plastic strain due to the dislocations related to the formation of s-th kind of variants in the training process is denoted by $\varepsilon_{s}^{\mathrm{cp}}$ (here "cp" represents "classical plasticity"). The corresponding macroscopic plastic strain $E_{s}^{c p}$ is proportional to the dislocation density which increases monotonically with the accumulated volume fraction $\mathrm{f}_{\mathrm{s}}^{\mathrm{ac}}$ (or number of cycling) of $\mathrm{s}$-th kind of martensite variant during the training process and finally reaches a saturation value. So the total plastic strain of the element can be expressed as

$$
\mathrm{E}^{p}=E^{t p}+E^{c p}=g \sum_{s=1}^{N} f_{s} \mathbf{R}_{s}+\sum_{s=1}^{N} f_{s}^{a c} \varepsilon_{s}^{c p} .
$$

Usually the magnitude of $E^{c p}$ is relatively small and can be considered as a constant after saturation.

\subsection{Free energy of the constitutive element}

\subsubsection{Elastic strain energy}

Both external and internal stress contribute to the elastic strain energy. The internal stress related elastic strain energy is due to phase transformation and dislocations. The calculation of the elastic strain energy for the above two kinds of contributions has been described in a unified manner in the book of Mura [12]. In the following we will consider the two contributions separately.

we first consider the elastic strain energy caused by pure phase transformation. Due to the incompatibility of the transformation strain of the variants with the surrounding elastic parent matrix, internal stress will be produced and elastic strain energy will be stored in the constitutive element. Many investigations show that such kind of elastic strain energy plays a very important role in the thermodynamics and kinetics of thermoelastic martensitic transformation. In order to calculate the elastic strain energy in the constitutive element, a concept of inclusion is used. A lot of micrographs show that a martensite variant appears as a shape of plate or blade, so here we approximate the geometric shape of variants by an oblate spheroid inclusion with $a_{1}=a_{2}, a_{3} / a_{1}=\rho<1$, where $a_{1}, a_{2}$ and $a_{3}$ are the principal half axes of the spheroid, and from experimental observations we can reasonably take the short axis of the spheroidal inclusion to be normal to the invariant plane of the variant. The value of $\rho$ can be different but here for simplicity all inclusions are assumed to have the same value of $\rho$ while the sizes and orientations of the inclusions may still be different. So different kinds of variants are represented by inclusions with different orientations of the short axes [3].

The equilibrium of constitutive element leads to the relation between microscopic stress $\sigma$ and macroscopic stress $\Sigma$,

$$
\Sigma=\langle\sigma\rangle_{V}=\frac{1}{V} \int_{V} \sigma d V=\sum_{s=1}^{N} f_{s}\langle\sigma\rangle_{V_{s}}+(1-f)\langle\sigma\rangle_{V_{p}} .
$$

By using micromechanics self-consistent approach (see SUN et al. [3]), the transformation-induced internal stress $\bar{\sigma}$ in the $s$ th $(s=1, \ldots, N)$ kind of inclusions can be expressed by

$$
\bar{\sigma}_{s}=\mathbf{L}:\left(\mathbf{S}_{s}-\mathbf{I}\right): \varepsilon_{s}^{t p}-\mathbf{L}: \sum_{i=1}^{N}\left(\mathbf{S}_{i}-\mathbf{I}\right): \varepsilon_{t}^{t p} f_{t} \text {, }
$$


where $\mathbf{L}=\mathbf{M}^{-1}$ is the elastic stiffness tensor of the material, $\mathbf{I}$ is the identity tensor and $S$ is the Eshelby tensor determined by elastic constants and the shape parameters (i.e. the orientation and the ratio $\rho$ ) of the oblate spheroidal inclusion. Obviously, $S$ is the same for inclusions of the same kind.

By Eq. (6b), the elastic strain energy $W^{t r}$ produced by transformation internal stress in a unit volume of the element can be calculated (MURA [12]) as

$$
W^{t r}=-\frac{1}{2 V} \int_{V_{i}} \bar{\sigma}: \varepsilon^{t p} d V=-\frac{1}{2} \sum_{s=1}^{N} f_{s} \varepsilon_{s}^{t p}: \mathbf{L}:\left(\mathbf{S}_{s}-\mathbf{I}\right): \varepsilon_{s}^{t p}+\frac{1}{2} \sum_{s=1}^{N} \sum_{t=1}^{N} f_{s} f_{t} \varepsilon_{s}^{t p}: \mathbf{L}:\left(\mathbf{S}_{t}-\mathbf{I}\right): \varepsilon_{t}^{t p}
$$

Defining

$$
\begin{aligned}
& W_{s}=-\frac{1}{2} \varepsilon_{s}^{t p}: \mathbf{L}:\left(\mathbf{S}_{s}-\mathbf{I}\right): \varepsilon_{s}^{t p}, \\
& W_{s t}=-\frac{1}{2} \varepsilon_{s}^{t p}: \mathbf{L}:\left(\mathbf{S}_{t}-\mathbf{I}\right): \varepsilon_{t}^{t p},
\end{aligned}
$$

we have

$$
W^{t r}=\sum_{s=1}^{N} f_{s} W_{s}-\sum_{s=1}^{N} \sum_{t=1}^{N} f_{s} f_{t} W_{s t} .
$$

Suppose in an infinite homogeneous medium stress-free at infinity there is one oblate spheroidal inclusion $I$ with eigenstrain $\varepsilon_{i}^{\mathrm{t}}$. According to Eshelby's solution, the internal stress in the inclusion is

$$
\bar{\sigma}_{i}^{\infty}=\mathbf{L}:\left(\mathbf{S}_{i}-\mathrm{I}\right): \varepsilon_{i}^{t p} \text {. }
$$

and the elastic strain energy induced by the internal stress in the infinite medium per unit volume of inclusion is

$$
\tilde{W}=-\frac{1}{2 V_{i}} \int_{V_{i}} \bar{\sigma}_{i}^{\infty}: \varepsilon_{i}^{i p} d V=-\frac{1}{2} \varepsilon_{i}^{t p}: \mathbf{L}:\left(\mathbf{S}_{i}-\mathbf{I}\right): \varepsilon_{i}^{t p} .
$$

Comparing (8) with (12), we can see that $W_{s}$ represents the elastic strain energy in an infinite medium due to a unit volume of oblate spheroidal inclusion with eigenstrain $\varepsilon_{\mathrm{s}}^{\mathrm{t}}$. In case of elastic isotropy we have $W_{s}=W_{t}(s, \mathrm{t}=1, \ldots, N)$. For simplicity, $W_{i}$ is used to replace $W_{s}$ in the following. So the first term in Eq. (10) can be written as $W_{i} \sum_{s=1}^{N} f_{s}$, which denotes the summation of the elastic strain energy due to each of the individual inclusions with the constitutive element being viewed as an infinite domain and without considering the interaction effects among those inclusions. The second term $\left(-\sum_{s=1}^{N} \sum_{t=1}^{N} f_{s} f_{t} W_{s t}\right)$ in Eq. (10) can be considered as a correction to the first term. For any two kinds of variants $s$ and $t$, we usually have (by Eq. (9))

$$
W_{s t} \neq W_{t s} \quad(s, t=1, \ldots, N)
$$

and when $s=t$, we have

$$
W_{s s}=W_{s}=W_{i}>0 \text {. }
$$

For convenience in mathematical treatment, we define a symmetrical matrix $\tilde{W}_{s t}$ as

$$
\tilde{W}_{s t}=\frac{1}{2}\left(W_{s t}+W_{s s}\right)
$$

then Eq. (10) becomes as

$$
W^{t r}=W_{i} \sum_{s=1}^{N} f_{s}-\sum_{s=1}^{N} \sum_{t=1}^{N} f_{s} f_{t} \tilde{W}_{s t} .
$$

If the constitutive element contains one kind of variants $s$ only, i.e., $f_{s}=f$, then according to Eqs (14), (15) and (16), we have $W^{t r}=W_{i} f(1-f)$ which vanishes for $f=0$ or $f=1$. 
Now we consider the elastic strain energy due to pure dislocations. The following simplifications are introduced: Because elastic strain energy of dislocation is proportional to the dislocation density which in turn increases proportionally with the accumulated volume fraction $\mathrm{f}_{\mathrm{s}}^{\mathrm{xc}}\left(=\int_{0}^{f_{5}}\left|d f_{s}\right|\right)$ until a saturation value is reached, so it can be approximately assumed that the stored elastic strain energy due to dislocations corresponding to the formation of the s-th kind of martensite variants is a monotonic increasing function of $f_{s}^{a c}$, i.e.,

$$
W_{s}^{d i s}=W_{s}^{d i s}\left(f_{s}^{a c}\right)
$$

The simplest form of which is

$$
W_{s}^{d i s}=D^{d i s} f_{s}^{a c}
$$

where $D^{d i s}$ is a material constant and can be understood as the energy density factor. So the total elastic strain energy due to dislocations can be expressed as

$$
W^{d i s}=\sum_{s=1}^{N} W_{s}^{d i s}=D^{d i s} \sum_{s=1}^{N} f_{s}^{a c}
$$

Here as an approximation the interaction between different kinds of dislocations are neglected.

Finally we consider the last and the most important interaction energy between stress fields of dislocation and transformation. Physically the appearance of favorable martensitic variants will relax the residual stress field of dislocation, which will reduce the stored elastic strain energy of the constitutive element. If the total stored elastic strain energy of the constitutive element consists three terms

$$
W=W^{t r}+W^{d i s}+W^{\text {int }}
$$

then the interaction energy $W^{\text {int }}$ has a negative value. By micromechanics analysis the main part of interaction energy can be generally written as

$$
W^{\mathrm{int}}=-H_{0} \sum_{s=1}^{N} f_{s}^{a c} \varepsilon_{s}^{c p} \sum_{t=1}^{N} f_{t} \varepsilon_{t}^{t p}
$$

where $H_{0}$ is a material constant. Detailed derivation of the above expression will be given elsewhere.

The total elastic strain energy $W$ per unit volume of the constitutive element can be expressed as (by using Eqs (16), (19), (20) and (21))

$$
W=\frac{1}{2} \Sigma: M: \Sigma+W_{i} \sum_{s=1}^{N} f_{s}-\sum_{s=1}^{N} \sum_{t=1}^{N} f_{s} f_{t} \tilde{W}_{s t}+D^{d i s} \sum_{s=1}^{N} f_{s}^{a c}-H_{0} \sum_{s=1}^{N} f_{s}^{a c} \varepsilon_{s}^{c p} \sum_{t=1}^{N} f_{t} \varepsilon_{t}^{r p}
$$

where the first term is the contribution due to applied stress. Here it must be noted that to obtain the total elastic strain energy by simply summation of three contributions is only for the simplicity of mathematical treatment. In fact there exists complicated interactions between the stress field of dislocations and the stress fields of transformations. In the present study, the interaction effects are taken into consideration in an aproximate manner.

\subsubsection{Surface energy and Chemical free energy}

The $p-m$ inter-phase surface energy in the constitutive element can be reasonably expressed as (see Fu, Muller and Xu [8])

$$
W_{\text {surf }}=A f(1-f)=A \sum_{i=1}^{N} f_{i}\left(1-\sum_{j=1}^{N} f_{j}\right),
$$

where $A$ is a constant related to the $p-m$ surface energy per unit area. The surface energy between martensite variants is ignored. The total change in chemical free energy $\Delta G_{\text {chem }}$ for unit volume of the constitutive element is

$$
\Delta G_{c h e m}(T)=\frac{\Delta G}{V} \sum_{s=1}^{N} V_{s}=\Delta G \sum_{s=1}^{N} f_{s}
$$


where $\Delta G=G_{m}(T)-G_{p}(T)$, and $G_{p}(T), G_{m}(T)$ are chemical free energy per unit volume of parent and martensite phase, respectively. Generally, $\Delta G$ can be approximated as a linear function of temperature $T$, i.e.

$$
\Delta G=k\left(T-T_{0}\right)
$$

where $T_{0}$ is the equilibrium temperature of the two phases and $k$ is a positive constant.

Now we can finally formulate the complementary free energy $\Psi$ of the constitutive element per unit volume as

$$
\begin{aligned}
& \Psi\left(\Sigma, T, f_{1}, \ldots, f_{N}, f_{1}^{a c}, \ldots, f_{N}^{a c}\right)=-\left(W+W_{\text {surf }}+\Delta G_{\text {chem }}-\Sigma: \mathrm{E}\right) \\
&= \frac{1}{2} \Sigma: \mathbf{M}: \Sigma+\Sigma: \sum_{s=1}^{N}\left(\varepsilon_{s}^{t p} f_{s}+f_{s}^{a c} \varepsilon_{s}^{c p}\right)-A \sum_{i=1}^{N} f_{i}\left(1-\sum_{j=1}^{N} f_{j}\right)- \\
&\left(W_{i}+\Delta G\right) \sum_{s=1}^{N} f_{s}+\sum_{s=1}^{N} \sum_{t=1}^{N} f_{s} f_{t} \tilde{W}_{s t}-D^{d i s} \sum_{s=1}^{N} f_{s}^{a c}+H_{0} \sum_{s=1}^{N} f_{s}^{a c} \varepsilon_{s}^{c p} \sum_{t=1}^{N} f_{t} \varepsilon_{t}^{t p}
\end{aligned}
$$

Here we can immediately see that $f_{1}, \ldots, f_{N}, f_{1}^{a c}, \ldots, f_{N}^{a c}$ are obviously the internal variables [14] describing the internal state of material as a results of loading history. $f_{1}, \ldots, f_{N}$ quantitatively characterize the pattern of microstructural rearrangement during pure transformation and reorientation and $f_{1}^{a c}, \ldots, f_{N}^{a c}$ represent averaged measures of the structural rearrangements due to dislocations.During the thermodynamic processes involving both transformation and reorientation, the second law of thermodynamics requires

$$
\left.\dot{\Psi}\right|_{\Sigma, T}=\sum_{s=1}^{N}\left(\frac{\partial \Psi}{\partial f_{s}} \dot{f}_{s}+\frac{\partial \Psi}{\partial f_{s}^{a c}} \dot{f}_{s}^{a c}\right)=\sum_{s=1}^{N}\left(F_{s} \pm F_{s}^{\prime}\right) \dot{f}_{s} \geq 0 .
$$

where the thermodynamic forces conjugate to the internal variables $f_{s}$ and $f_{s}^{a c}$ are denoted by $F_{s}$ and $F_{s}$ respectively. Since thermodynamic forces depend on the value of $f_{1}, \ldots, f_{N}$ (state variables), it is seen that energy dissipation rate is a function of internal state variable (In section 2.3 below an energy dissipation rate function will be formulated). Generally, the variation of the volume fraction of $s$ th $(s=1, \ldots, N)$ kind of variants has two origins: one is the transformation processes between the sth kind of variants and the parent phase, i.e., the forward and reverse transformations, the other is the reorientation processes between the sth kind of variants and the rest kinds of variants. Denoting time rate $d(\quad) / d t$ by ( ), the volume fraction rate of the sth kind of variants can be expressed as

$$
\dot{f_{s}}=\dot{f}_{s 0}+\dot{f}_{s 1}+\cdots+\dot{f}_{s s-1}+\dot{f}_{s s+1}+\cdots+\dot{f}_{s N} \quad(s=1, \ldots, N) .
$$

where $\dot{f}_{s 0}$ represents the part of the volume fraction rate caused by the forward or reverse transformations. $\dot{f}_{s t}(t=1, \ldots, s-1, s+1, \ldots, N)$ in Eq.(28) represents the part of the volume fraction rate caused by the reorientation process. $\dot{f}_{s t}>0$ means that some part of the $t$ th kind of variants is reoriented to the $s$ th kind of variants and vice versa. According to the definition of reorientation, we have

$$
\dot{f}_{s t}=-\dot{f}_{t s} \quad(s=1, \ldots N ; t=1, \ldots s-1, s+1, \ldots, N) .
$$

\subsection{Analysis of energy dissipation during transformation and reorientation}

The total energy dissipation $W_{d}$ (or the energy dissipation rate $\dot{W}_{d}$ ) of the material can be considered as the sum of the energy dissipation (or energy dissipation rate) of different kinds of variants which comes from: (1) the forth and back motion of the parent and martensite (p-m) interface; (2) the inter-variant boundary motion during the reorientation process. Recent experimental studies [13] have revealed some features of the energy dissipation, the most important of which is the discovery of the internal hysteresis loops, i.e. the previous transformation history effect on the energy dissipation. Though some theoretical 
investigations have been performed on this kind of phenomena, the results so far obtained are not satisfactory.

In this section, we give a quantitative discription of the history effect. The description is based on the energy dissipation analysis of one kind of variant during pseudoelasticity, and then to use this relation to obtain a generalized energy dissipation expression.

We first conjecture that energy dissipation rate $\dot{W}_{d}^{t r-x}$ of the sth kind of variant is proportional to the rate of its volume fraction $\dot{f}_{s 0}$ (during forward and reverse transformations)

$$
\dot{W}_{d}^{t r-s}=D_{d}^{t r} \dot{f}_{s o}
$$

where $D_{d}^{t r}$ is the proportionality. We second conjecture that $D_{d}^{t r}$ not only depends on the current value of the volume fraction of the sth kind of martensite variant (such as implied by Eqs.(26) and (27)) but also depends on the value of the volume fraction of the martensite just before the variant changes its direction of the transformation process (such as change from farward transformation to reverse transformation), this volume fraction is termed as the memory volume fraction and is denoted by $f_{s}^{\text {mem }}$, i.e.,

$$
D_{d}^{t r}=D_{d}^{t r}\left(f_{s}, f_{s}^{\text {mem }}\right) \text {. }
$$

The simplest function form of $\mathrm{D}_{\mathrm{d}}^{\mathrm{t}}$ is

$$
D_{d}^{t r}= \pm D_{0}^{d r}+\alpha^{t r}\left(f_{s}-f_{s}^{\text {mem }}\right),
$$

where $D_{0}^{t r}$ and $\alpha^{\text {tr }}$ are positive material constants (their physical meaning will be explained in the following), + and - corresponds to the forward and reverse transformation process respectively. Here it is noted that one expression represents two processes $(\mathrm{p} \rightarrow \mathrm{m}$ and $\mathrm{m} \rightarrow \mathrm{p})$. The energy dissipation rate in general case can, by extension, be written as

$$
\dot{W}_{d}=\dot{W}_{d}^{t r}+\dot{W}_{d}^{r e}=\sum_{s=1}^{N} \dot{W}_{d}^{t r-s}+\frac{1}{2} \sum_{s=1}^{N} \sum_{\substack{t=1 \\ \imath \neq s}}^{N} \dot{W}_{d}^{r e-s t},
$$

where

$$
\begin{aligned}
& \dot{W}_{d}^{r e-s t}=D_{d}^{r e-s t} \dot{f}_{s t}, \\
& D_{d}^{r e-s t}= \pm D_{0}^{r e}+\alpha^{r e}\left(f_{s}-f_{s}^{m e m}\right),
\end{aligned}
$$

where + and - corresponds to the $t \rightarrow s$ and $s \rightarrow t$ reorientation processes, respectively. The above expressions agree well with the available exprimental data (MÜLLER and XU [8]).

\subsection{Constitutive laws for transformation and reorientation in stress space}

\subsubsection{Transformation and reorientation (yielding) conditions}

In stress space, the thermodynamic forces conjugate to the internal variable $f_{s}$ and $\mathrm{f}_{\mathrm{s}}^{\mathrm{ac}}$ are

$$
\begin{aligned}
& F_{s}=\frac{\partial \Psi}{\partial f_{s}}=\varepsilon_{s}^{t p}: \Sigma+2 \sum_{t=1}^{N} \tilde{W}_{s s} f_{t}-\left(W_{i}+\Delta G\right)-A\left(1-2 \sum_{i=1}^{N} f_{i}\right)+H_{0} \varepsilon_{s}^{t p} \sum_{t=1}^{N} f_{t}^{a c} \varepsilon_{t}^{c p}, \\
& F_{s}^{\prime}=\frac{\partial \Psi}{\partial f_{s}^{a c}}=H_{0} \varepsilon_{s}^{c p} \sum_{t=1}^{N} f_{t} \varepsilon_{t}^{t p}+\Sigma: \varepsilon_{s}^{c p}-D^{d i s}
\end{aligned}
$$

With the change of the applied thermodynamic conditions, the volume fractions of all kinds of variants in the constitutive element are possible to change, during which $\left.\dot{\Psi}\right|_{\Sigma, T}$ must be equal to the energy dissipation rate $W_{d}$ (according to thermodynamics), i.e.,

$$
\sum_{s=1}^{N}\left(F_{s} \pm F_{s}^{\prime}\right) \dot{f}_{s}=\sum_{s=1}^{N} D_{d}^{t r} \dot{f}_{s o}+\frac{1}{2} \sum_{s=1}^{N} \sum_{\substack{t=1 \\ t \neq s}}^{N} D_{d}^{r e-s t} \dot{f}_{s t}
$$


Substituting Eq. (28) into Eq. (38) we obtain:

$$
\sum_{s=1}^{N}\left(F_{s} \pm F_{s}^{\prime}-D_{d}^{t r}\right) \dot{f}_{s 0}+\sum_{s=1}^{N} \sum_{\substack{t=1 \\ t \neq s}}^{N}\left(F_{s}-F_{t} \pm\left(F_{s}^{\prime}-F_{t}\right)-D_{d}^{r e-s t}\right) \dot{f}_{s t}=0 \text {. }
$$

Because it is suitable to any possible combinations of $\left(\dot{f}_{10}, \ldots, \dot{f}_{N 0}, \ldots, \dot{f}_{1 N}, \ldots, \dot{f}_{N-1 N}\right)$, we must have

$$
\left\{\begin{array}{c}
F_{s} \pm F_{s}^{i}-D_{d}^{t r}=0 \\
F_{s}-F_{t} \pm\left(F_{s}-F_{t}\right)-D_{d}^{r e-s t}=0
\end{array} \quad(s=1, \ldots, N ; t=1, \ldots, s-1, s+1, \ldots, N),\right.
$$

Substituting Eqs. (36) and (37) into Eq. (40), we obtain the transformation yield conditions $Y_{\text {so }}$ for the $s$ th $(s=1, \ldots, N)$ kind of martensite variants and the reorientation conditions $Y_{s t}$ for the th $(t=1, \ldots, s-1, s+1, \ldots, N)$ kind of variants to be reoriented to the $s$ th $(s=1, \ldots, N)$, which are expressed by $Y_{s 0}\left(\Sigma, T, f_{1}, \ldots, f_{N}, f_{1}^{a c}, \ldots, f_{N}^{a c}\right)=$

$\Sigma:\left(\varepsilon_{s}^{t p} \pm \varepsilon_{s}^{c p}\right)+2 \sum_{i=1}^{N} \tilde{W}_{s t} f_{t}-\left(W_{i}+\Delta G\right)-A\left(1-2 \sum_{i=1}^{N} f_{i}\right)+H_{0} \varepsilon_{s}^{t p} \sum_{i=1}^{N} f_{t}^{a c} \varepsilon_{t}^{c p} \pm H_{0} \varepsilon_{s}^{c p} \sum_{i=1}^{N} f_{i} \varepsilon_{t}^{t p} \mp D^{d i s}-D_{d}^{d r}=0$

$Y_{s t}\left(\Sigma, T, f_{1}, \ldots, f_{N}, f_{1}^{a c}, \ldots, f_{N}^{a c}\right)=$

$\Sigma:\left(\left(\varepsilon_{s}^{t p} \pm \varepsilon_{s}^{c p}\right)-\left(\varepsilon_{t}^{t p} \pm \varepsilon_{t}^{c p}\right)\right)+2 \sum_{n=1}^{N}\left(\tilde{W}_{s n}-\tilde{W}_{t n}\right) f_{n}+H_{0}\left(\varepsilon_{s}^{t p}-\varepsilon_{t}^{t p}\right) \sum_{k=1}^{N} f_{k}^{a c} \varepsilon_{k}^{c p} \pm H_{0}\left(\varepsilon_{s}^{c p}-\varepsilon_{t}^{c p}\right) \sum_{k=1}^{N} f_{k} \varepsilon_{k}^{t p}-D_{d}^{r e-s t}=0$

$$
(s=1, \ldots, N ; t=1, \ldots, s-1, s+1, \ldots, N) \text {. }
$$

In the above two equations, " + " and "-" in " \pm " is for the forward and reverse transformation respectively. The incremental form of the constitutive relations can be derived by the normality rule and consistency condition. The detailed formulation has been described in the recent paper by Sun et al.[3].

\subsubsection{Some discussions}

From the derived transformation (yielding) conditions (Eq.(41) and (42)) it is seen that: (1) if there is no training, i.e., $f_{s}^{a c}=0(\mathrm{~s}=1,2, \ldots, \mathrm{N})$, these yielding conditions turns exactly to be that of OWSME (see Sun et al.[3]). This means that the present yielding condition is a more generalised version for the constitutive description of shape memory alloys. (2) after the training process with the formation of a particular kind of martensite variants (as in the case of thermal cycling training with constant applied stress), the stress needed to induce this particular type of variant will be smaller than the case without training, and in the case of pure thermal cycling this type of particular martensite variants will appear at higher temperature than the case without training and growth continuously thus causing the observed TWSME. (3) after the training process with pure thermal cycling without applied stress(i.e., the spontaneous formation of 24 kinds of martensite variants), the transformation stresses needed to induce any kind of variant will be smaller than the case without training, and in the case of subsequent pure thermal cycling the $M_{s}$ temperature will be higher than the case without training(i.e., a shift in $M_{s}$ ).

The above qualitative preditions of the model are all in agreement with the experimental observations. Detailed quatitative comparison between theory and experiments will be given in another paper.

\section{CONCLUSION}

Based on micromechanics, crystallographic theory and the analysis of interaction between stress fields of dislocation and transformation, a constitutive model to describe both one-way shape memory effect (OWSME) and two-way shape memory effect (TWSME) phenomena of single crystal during thermoelastic martensitic transformation is proposed. The history effect of the previous training process (i.e., the effect of dislocations) on the constitutive behavior is described by the accumulated martensitic variant volume 
fractions. The nonproportional loading history effect of pure transformation plasticity on the constitutive behavior is described by the current values of the volume fraction of martensite variants. The important interaction between the stress fields of dislocations and phase transformations has been taken into consideration. This constitutive model is a more generalised one in the sense that it makes the constitutive description of both OWSME and TWSME phenomena of single crystals in an unified interrelated mannar and is applicable under complex thermomechanical loading conditions. At the same time, it can be used as a theoretical basis to predict the OWSME and TWSME of polycrystals.

\section{Acknowledgements}

The authors are sincerely grateful to the National Natural Science Foundation of China and the French Ministry of Education for their support.

\section{References}

[1] Stalmans R., van Humbeeck J. and Delaey L., J. de Physiqu IV, Colloque C4, supplement au J. de Physique III, Vol.1(1991) C4-403--C4-408.

[2] Cesari E., Picornell C., Pons J. and Sade M., J. de Physiqu IV, Colloque C4, supplement au J. de Physique III, Vol. 1 (1991) C4-451--C4-456.

[3] Sun, Q. P., Yan, W. Y. and Hwang, K. C., 1994, submitted to the J. Mech Phys. Solids for publication.

[4] Patoor, E., Eberhardt, A. and Berveiller, M., Acta metall., 35 (1987) 2779-2789.

[5] Sun, Q. P., Hwang, K. C. and Yu, S. W., J. Mech. Phys. Solids, 39 (1991) 507-524.

[6] Sun, Q. P., and Hwang, K. C., J. Mech. Phys. Solids, 41(1993) 1-33.

[7] Sun, Q. P., and Hwang, K. C., Advances in Applied Mechanics, Vol.31, Academic Press, New York, 1994, pp.249-298.

[8] Muller, I. and Xu, H., Acta Metall. Mater., 39 (1991) 263-271.

[9] Tanaka, K., Kobayashi, S. and Sato, Y., Int. J. Plast., 2(1986), 59-72.

[10]Abeyaratne, R. and Knowles, J. K., J. Mech. Phys. Solids, 41(1993), 541-560.

[11] Patoor, E., Barbe, P., Eberhardt, A. and Berveiller, M., J. de Physiqu IV, Colloque C4, supplement au J. de Physique III, Vol.1(1991) C4-95--C4-100.

[12] Mura, T., Micromechanics of Defects in Solids, Martinus Nijhoff, Dordrecht, 1987.

[13] Fu, S., Muller, I. and Xu, H., "On the thermodynamics of pseudoelasticity", In Proceedings of the International Conference on Martensitic Transformations, 1992, Monterey, CA (USA), pp 335-340.

[14] Rice J. R., J. Mech. Phys. Solids, 19 (1971) 433-455. 